\title{
UN NOUVEL ACARE DE LA GALE DANS L'EX CONGO BELGE
}

\author{
Par R. F. LAWRENCE
}

Dans une importante collection d'Acariens montés, envoyée par le $\mathrm{D}^{\mathrm{r}}$ H. G. Vercammen-Grandjean, de Bukavu (Congo belge), nous avons découvert une préparation contenant quatre femelles de Sarcoptidés, avec un certain nombre d'œufs encapsulés dans la peau superficielle. Ces Acares provenaient d'un rat des marais ou des « criques 》, Pelomys fallax, l'une des multiples espèces de Rongeurs sauvages trouvées au Congo, et dont la très large distribution géographique s'étend de l'Est africain portugais et de l'Angola au Sud, jusqu'aux Congo belge, Tanganyika, Kenya, et à l'Uganda.

Bien qu'appartenant à la famille des Sarcoptidæ, cet Acare ne s'identifie parfaitement à aucun des six genres (1) qui composent actuellement cette famille, et un genre nouveau est ici proposé pour le désigner.

\section{Genre Mysarcoptes nov. gen.}

Présente les caractères génériques de Sarcoptes, Notoedres et Trixacarus pour la disposition des soies des pattes et des structures apicales des tarses chez la femelle, mais se distingue de ces trois genres (exception faite du cas de Notoedres muris) par des soies dorsales plus grêles et moins nombreuses.

Se rapproche de Notoedres par les soies verticales antérieures du propodosome, très petites et fines, et par l'absence de bouclier dorsal bien défini ; mais s'en écarte notablement par la position terminale et non dorsale de l'anus. Par ce dernier caractère, ressemble à Sarcoptes et Trixacarus, mais s'en écarte par la forme distincte et caractéristique de l'orifice anal (fig. $3 \mathrm{~b}$ ) ; diffère de Trixacarus par l'absence de bouclier polygonal chitineux, celui-ci étant petit, mal défini et situé seulement un peu en avant de la moitié du corps.

ESPÈCE TYPE : Mysarcoptes paucipllis nov. sp.

(1) Baker et Wharton (1952) en reconnaissaient sept, mais Teinocoptes a été retiré par Fain (1959) pour servir de base à une nouvelle famille, les Teinocoptidæ. 


\section{MYSARCOPTES PAUCIPILIS nov. sp.}

Holotype: Une femelle de Pelomys fallax, Bukavu, Congo belge, récoltée par H.-G. Vercammen-Grandjean ; paratypes : trois femelles du même hôte.

Face dorsale (fig. 1) : Très fines et légères striations, le bouclier propodosomal mal défini, à limite antérieure indistincte et située près du milieu du corps avec une petite soie près de chacun des

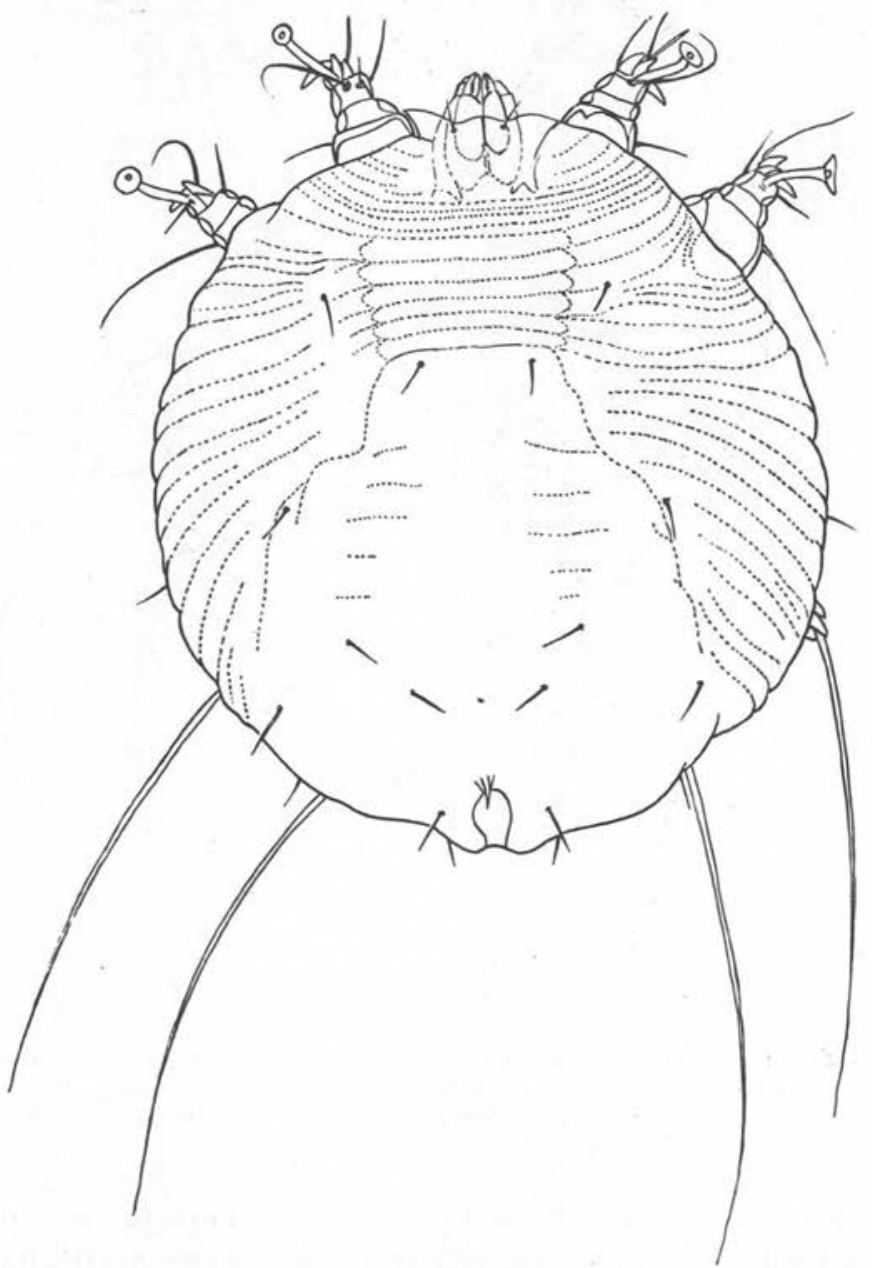

FIG. 1. - Mysarcoptes paucipilis gen. ei sp. nov. Holotype $₹$; face dorsale 
angles antéro-latéraux; sept paires de soies fines et courtes en sus d'une paire de petites soies verticales antérieures, la majorité d'entre elles situées dans une aire occupant les deux tiers-postérieurs

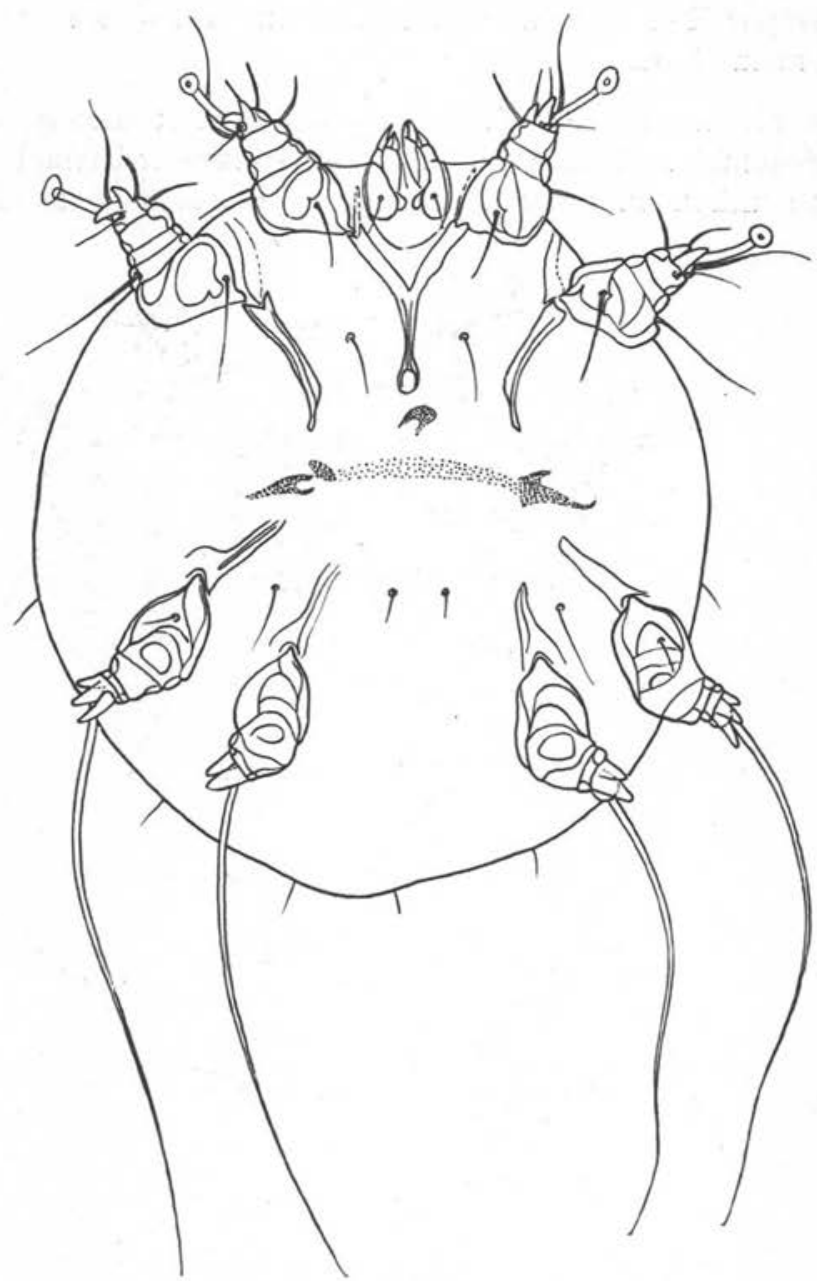

Fig. 2. - Mysarcoptes paucipilis sp. nov. Paratype $\subsetneq$; face ventrale. Les épimères et les segments basaux des pattes, très chitinisés, jaune-brun, contrastent nettement avec les parties non chitinisées.

de la moitié inférieure de la face dorsale, laquelle est presque complètement dépourvue de striations. Les soies verticales antérieures plus courtes et plus fines que la moyenne des soies dorsales, situées plus en avant que chez Sarcoptes, Notoedres et Trixacarus, juste derrière le bord antérieur du propodosome. 
Anus s'ouvrant à l'extrémité dorsale postérieure, dans une concavité piriforme aux bords épaissis, plus opaques que les parties voisines; la forme de l'ouverture très distincte, identique chez les
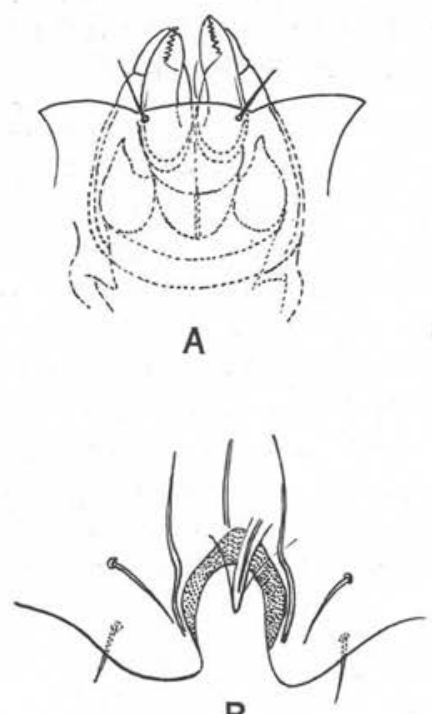

B

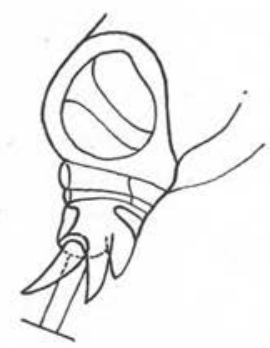

$E$

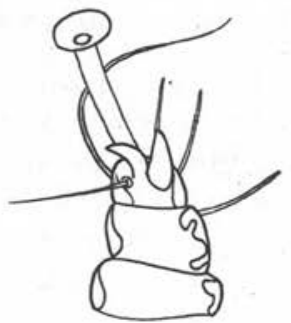

C

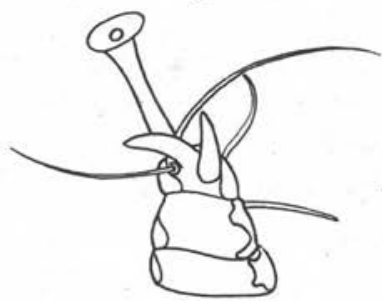

D

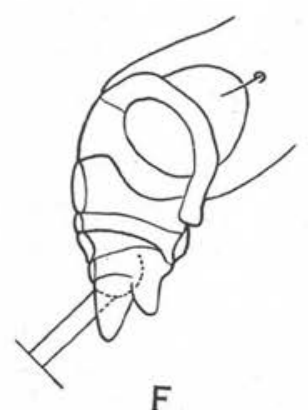

FıG. 3. - Mysarcoptes paucipilis sp. nov. Paratype + ; A) Pièces buccales, vue dorsale; B) Ouverture anale, vue dorsale ; C) Pattes I, vue ventrale; D) Pattes II, vue ventrale ; E) Pattes III, vue ventrale; F) Pattes IV, vue ventrale.

quatre femelles, et différente de celles des autres formes connues de Sarcoptidæ (fig. $3 b$ ).

Face ventrale (fig. 2) : Représentant un des paratypes femelles ; pore génital s'ouvrant juste au-dessous du sternum médian en forme d'Y, très petit, quoique fortement chitinisé ; de chaque côté, 
entre l'épimère des $2^{e}$ et $3^{e}$ paires de pattes, une structure chitineuse aux contours indécis.

Pièces buccales (fig. $3 a$ ) : Grands chélicères et pédipalpes bien développés, non masqués par le bord antérieur du propodosome comme chez beaucoup d'autres genres de Sarcoptidés.

Pattes : Les deux paires antérieures avec tarses en forme de cloche, et de taille égale ; I et II (fig. $3 c$ et $d$ ) se terminant par trois sortes de griffes (la troisième griffe supérieure, courte, non visible sur la fig. $3 c$ et $d$ ) ; des deux griffes inférieures, la plus postérieure s'insère presqu'à angle droit avec la griffe antérieure qui est orientée directement en avant ; la griffe postérieure de II nettement plus longue que celle de I. Segment basal des pattes antérieures portant une soie fine, celle de II deux fois plus longue que celle de I; III avec trois griffes coniques à l'apex (la griffe basale plus courte et obtuse) ; IV présentant deux griffes seulement (fig. $3 e, f$ ); le long flagelle simple terminal des tarses III et IV de taille sensiblement égale, nettement plus court que la longueur du corps, légèrement inférieur à sa largeur.

Dimensions : Femelle type ; longueur du corps : $300 \mu$; largeur : $262 \mu$. Paratype femelle : $250 \mu$ et $218 \mu$ respectivement.

Un certain nombre d'œufs avec quelques poils in situ ont été montés. Ils semblent encastrés avec les poils dans la peau.

Les œufs contiennent des larves en voie de développement à différents stades.

La structure des plus avancées d'entre elles se discerne aisément. Aucune des plus petites soies dorsales et ventrales n'est présente ; mais les pièces buccales, les épimères et trois paires de pattes bien développées sont clairement visibles, les épimères de toutes les pattes se rejoignant presqu'au milieu de la face ventrale. Les pattes de la $3^{\circ}$ paire présentent trois et non deux éperons coniques, en forme de griffe à leur extrémité, et un long flagelle simple comme chez l'adulte, replié à l'intérieur et s'entrecroisant.

L'une des quatre femelles adultes ne présentait qu'un seul œuf, similaire par la taille et la forme à ceux ci-dessus décrits, mais sans structure en voie de développement à son intérieur.

Pas de nymphes, ni de mâles présents.

\section{Discussion}

Comme nous l'avons indiqué dans la diagnose du groupe, cette forme se rapproche de trois genres de Sarcoptidæ: Sarcoptes, Notoedres et Trixacarus. 
Les espèces de Notoedres et Trixacarus (mais non, semble-t-il, de Sarcoptes) ont été trouvées sur des Rongeurs, et, dans les deux cas, chez le surmulot Rattus norvegicus.

L'Acare que nous décrivons ici diffère sensiblement de Sarcoptes par la disposition des soies sur la face dorsale; dans ce dernier genre, les soies verticales antérieures et les soies dorsales de l'opisthosome sont toutes de grosses épines épaisses, à extrémité obtuse, mélangées à de petits spicules pointus, tandis que celles de Mysarcoptes sont courtes, minces, difficiles à voir. Chez Sarcoptes aussi, le bouclier dorsal est clairement défini, placé très en avant et flanqué à chaque angle postéro-latéral d'une soie très longue et très pointue. Les striations dorsales sont beaucoup plus nombreuses et plus nettement définies chez Sarcoptes que chez Mysarcoptes.

Mysarcoptes ressemble à Notoedres par la disposition et le petit nombre de soies dorsales qui, dans ce genre, sont aussi grêles, ainsi que par ses minuscules soies antérieures verticales. Il diffère de Notoedres, et en particulier de l'espèce parasite de Rongeurs, $\mathrm{N}$. muris, par une configuration tout à fait différente des striations comportant notamment une partie médiane presque dépourvue de stries ; la portion antérieure de celle-ci pourrait être interprétée comme le bouclier, structure totalement absente chez Notoedres; les pattes sont plus grandes et mieux développées que chez Notoedres; les soies des pattes postérieures sont plus longues que celles de $N$. muris. Une autre différence réside dans la position de l'orifice anal, nettement situé sur la face dorsale chez Notoedres, c'est-à-dire franchement au-dessus du bord postérieur du corps, et par ailleurs différemment constitué.

Mysarcoptes se distingue de Trixacarus Sellnick (1944) par l'absence de bouclier chitineux et de longues soies et spicules sur la face dorsale ; il s'en écarte, en outre, par le détail des tarses des pattes III et IV qui présentent trois au lieu de deux griffes en éperon à l'apex de III, et par l'absence d'un poil accessoire à la base du long flagelle simple sur les tarses III et IV, qui est caractéristique de Trixacarus.

\section{Bibliographie}

BaKer (E. W.) and Wharton (G. W.), 1952. - An introduction to Acarology, New-York, Macmillan.

FAIN (A.), 1959. - Les Acariens psoriques parasites des Chauves-Souris : IV. Le genre Teinocoptes Rodhain. Création d'une nouvelle famille Teinocoptidæ fam. nov. (Sarcoptiformes). Rev. Zool. Bot. Afr., 68.

SELLNICK (M.), 1944. - Eine neue parasitische Milbe von Epimys norvegicus. Zeitschr. f. Parasitenkunde, 13 (3), 248.

Natal Museum - Pietermaritzbourg - Afrique du Sud 